\title{
Bernstein Series Solution of the Heat Equation in 2-D
}

\author{
Nurcan Baykuş Savaşaneril \\ Izmir Vocational School, Dokuz Eylül University, Izmir, Turkey
}

Received: 20 October 2017, Accepted: 15 November 2017

Published online: 12 December 2017.

\begin{abstract}
A broad class of steady-state physical problems can be reduced to finding the harmonic functions that satisfy certain boundary conditions. The Dirichlet problem for the Laplace equation is one of the above mentioned problems. In this paper, a numerical matrix method is developed for numerically solving the Heat equation in 2-D. The method converts the heat equation in 2-D to a matrix equation, which corresponds to a system of linear algebraic equations. Error analysis is included to demonstrate the validity and applicability of the technique. Finally, the effectiveness of the method is illustrated in the heat equation for a cut ring region.
\end{abstract}

Keywords: Heat equation, collocation method; Bernstein series solution; error analysis.

\section{Introduction}

Today, the theory of complex variables is used to solve problems of heat flow, fluid mechanics, aerodynamics, electromagnetic theory and practically every other field of science and engineering. A broad class of steady-state physical problems can be reduced to finding the harmonic functions that satisfy certain boundary conditions. The Dirichlet problem is to find a function $U(z)$ that is harmonic in a bounded domain $D \subset R^{2}$, is continuous up to the boundary $\partial D$ of $D$, assumes the specified values $U_{0}(z)$ on the boundary $\partial D$, where $U_{0}(z)$ is a continuous function on $\partial D$. Let $D$ be a rectangular region and $\partial D$ is the boundary of $D$ and can be formulated as

$$
\nabla^{2} U=0, \quad z \in D,\left.\quad U\right|_{z \in \partial D}=U_{0}(z)
$$

Here, for a point $(x, y)$ in the plane $R^{2}$, one takes the complex notation $z=x+y i, U(z)=U(x, y)$ and $U_{0}(z)=U_{0}(x, y)$ are real functions and $\nabla^{2} U=\frac{\partial^{2}}{\partial x^{2}}+\frac{\partial^{2}}{\partial y^{2}}$ is the Laplace operator. Similarly the Dirichlet problem for the Poisson equation can be formulated as

$$
\nabla^{2} U=h(z), \quad z \in D,\left.U\right|_{z \in \partial D}=U_{0}(z)
$$

The Green function of the Dirichlet problem for the Laplace differential equation in a triangle region was expressed in terms of elliptic functions and the solution of problem was based on the Green function, and therefore on elliptic functions by Kurt and Sezer [9,10]. Solution of the two-dimensional heat equation in a square region was given by Kurt [11]. Analytic solution was given two-dimensional heat equation for some regions by Baykuş Savaşaneril et al. [2,3,5,7]. The Chebyshev tau technique for the solution of Laplace's equation [1] and Chebyshev tau matrix method for Poisson-type equations in irregular domain [8] were studied by Ahmadi et al and Kong et al. Error analysis of the Chebyshev collocation method for linear second-order partial differential equations was expressed by Yüksel et al. $[13,14,15]$. 
In this paper, we have solved a matrix method which is based on Bernstein polynomials and collocation points. Let we consider the Dirichlet problem for the Poisson equation on $D=[0, a] \times[0, b]$ can be formulated as

$$
\nabla^{2} U=G(x, y)
$$

with the conditions defined at the points $x=\alpha_{k}, y=\beta_{k}$

$$
\sum_{k=1}^{t} \sum_{i=0}^{1} \sum_{j=0}^{1} a_{i, j}^{k} U^{(i, j)}\left(\alpha_{k}, \beta_{k}\right)=\lambda_{t}
$$

for $\left(\alpha_{k}, \beta_{k}\right) \in \partial D, a_{i, j}^{k}, k=1, \ldots, t$ and $\lambda_{t}$ are constants. Here $G(x, y)$ are functions defined on $D$. We will find an approximated solution, namely Bernstein series solution, of (1) such that

$$
U_{n, n}(x, y)=\sum_{i=0}^{n} \sum_{j=0}^{n} a_{i j} \mathbf{B}_{i, n}(x) \mathbf{B}_{j, n}(y)
$$

where $\mathbf{B}_{k, n}, 0 \leq k \leq n$ are Bernstein polynomials.

In this study, we will find a matrix form for each term in (1). Substituting these matrix forms into (1) gives a matrix equation fundemental matrix equation. We then obtain a linear system by using collocation points. Moreover, this operation gives a new approximate solution.

\section{The conform mapping of a cut-ring domain}

The conformal mapping of the cut-ring in the $z$-plane, onto the rectangle in the $w$-plane can be written as [3].

$$
w=\log z
$$

which is illustrated in Fig. 1. [4]

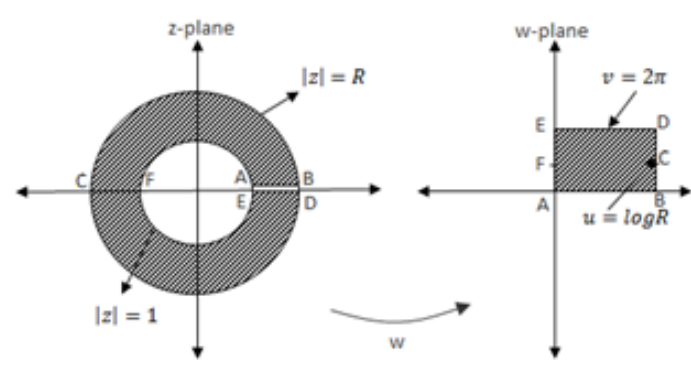

Fig. 1: Conformal mapping of the cut-ring in the $z$-plane onto the rectangular in the $w$-plane

Now let the function $t=\log z$, with $1 \leq|z| \leq 10^{2 K} ; k=3-2 \sqrt{2} ; K^{\prime}=2 K ; K^{\prime} \simeq \pi$ [12], maps the cut-ring onto the rectangle of the $t$-plane maps the rectangle with $0<\operatorname{Re}(t)<2 K, 0<\operatorname{Im}(t)<2 K^{\prime}$ onto the rectangle which is seen Fig. 2 


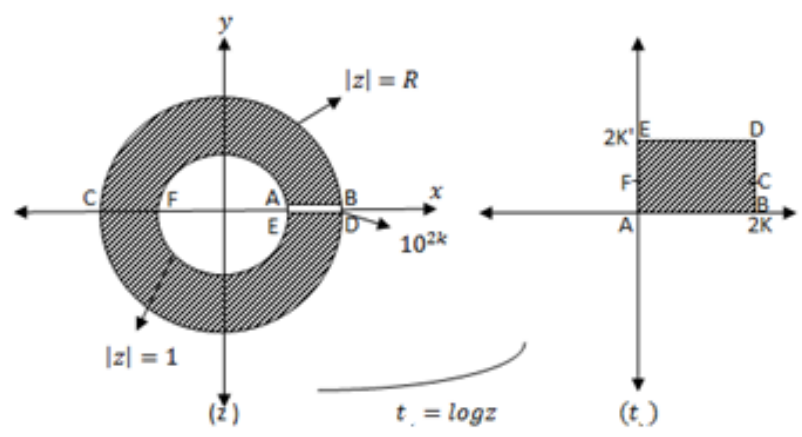

Fig. 2: Conformal mapping of the cut-ring in the $z$-plane onto the rectangular in the $w$-plane

\section{Fundamental relation}

Let $U_{n, n}$ be Bernstein series solution of (1). Also Let us find the matrix form of $U_{n, n}$ and $U_{n, n}^{(i, j)}=\frac{\partial^{i+j} U_{n, n}}{\partial x^{i} \partial y^{j}}$. $U_{n, n}$ can be written as

$$
U_{n, n}(x, y)=\mathbf{B}_{n}(x) \mathbf{Q}_{n}(y) \mathbf{A}
$$

where

$$
\begin{aligned}
& \mathbf{B}_{n}(x)=\left[B_{0, n}(x) B_{1, n}(x) \cdots B_{n, n}(x)\right] \\
& \mathbf{Q}_{n}(y)=\left[\begin{array}{cccc}
B_{n}(y) & 0 & \cdots & 0 \\
0 & B_{n}(y) & \cdots & 0 \\
\vdots & \vdots & \ddots & \vdots \\
0 & 0 & \cdots & B_{n}(y)
\end{array}\right]
\end{aligned}
$$

and

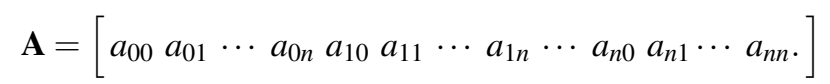

Therefore, $U_{n, n}^{(i, j)}$ can be written as

$$
U_{n, n}^{(i, j)}(x, y)=\mathbf{B}_{n}^{i}(x) \mathbf{Q}_{n}^{j}(y) \mathbf{A} .
$$

on the other hand, $\mathbf{B}_{n}^{(i)}(x)$ can be written as [6]

$$
\mathbf{B}_{n}^{i}(x)=\mathbf{X}^{(i)}(x) \mathbf{D}^{T}
$$

where

$$
\begin{aligned}
& \mathbf{D}=\left[\begin{array}{cccc}
d_{00} & d_{01} & \cdots & d_{0 n} \\
d_{10} & d_{11} & \cdots & d_{1 n} \\
\vdots & \vdots & \ddots & \vdots \\
d_{n 0} & d_{n 1} & \cdots & d_{n n}
\end{array}\right], d_{i j}= \begin{cases}\frac{(-1)^{j-i}}{R^{j}}\left(\begin{array}{l}
n \\
j
\end{array}\right)\left(\begin{array}{c}
n-i \\
j-i
\end{array}\right), & i \leq j \\
0, & i>j\end{cases} \\
& \mathbf{X}(x)=\left[\begin{array}{lll}
1 & \cdots & \cdots \\
x^{n}
\end{array}\right]
\end{aligned}
$$

for $\mathbf{X}^{(i)}(x)$, the relation

$$
\mathbf{X}^{(k)}=\mathbf{X}(x) \mathbf{B}^{k}
$$


is obtained where

$$
\mathbf{B}=\left[\begin{array}{cccccc}
0 & 1 & 0 & 0 & \cdots & 0 \\
0 & 0 & 2 & 0 & \cdots & 0 \\
0 & 0 & 0 & 3 & \cdots & 0 \\
\vdots & \vdots & \vdots & \vdots & \ddots & \vdots \\
0 & 0 & 0 & 0 & \cdots & n \\
0 & 0 & 0 & 0 & \cdots & 0
\end{array}\right]
$$

Substituting (6) into (5) yields

$$
\mathbf{B}_{n}^{(i)}=\mathbf{X}(x) \mathbf{B}^{i} \mathbf{D}^{T}
$$

If a similar procedure is carried out, $\mathbf{Q}_{n}(y)$, the relation $\mathbf{Q}_{n}(y)=\overline{\mathbf{Y}}(y) \overline{\mathbf{D}}$ will be obtained where

$$
\begin{gathered}
\overline{\mathbf{Y}}(y)=\left[\begin{array}{cccc}
Y(y) & 0 & \cdots & 0 \\
0 & Y(y) & \cdots & 0 \\
\vdots & \vdots & \ddots & \vdots \\
0 & 0 & \cdots & Y(y)
\end{array}\right], \quad \mathbf{Y}(y)=\left[\begin{array}{llll}
1 & y & \cdots & y^{n}
\end{array}\right] \\
\overline{\mathbf{D}}=\left[\begin{array}{cccc}
D^{T} & 0 & \cdots & 0 \\
0 & D^{T} & \cdots & 0 \\
\vdots & \vdots & \ddots & \vdots \\
0 & 0 & \cdots & D^{T}
\end{array}\right]
\end{gathered}
$$

Thus, $\overline{\mathbf{Y}}^{(j)}(y)$ can be written as

$$
\overline{\mathbf{Y}}^{(j)}(y)=\overline{\mathbf{Y}}(y) \overline{\mathbf{B}}^{j}
$$

where

$$
\overline{\mathbf{B}}=\left[\begin{array}{cccc}
B & 0 & \cdots & 0 \\
0 & B & \cdots & 0 \\
\vdots & \vdots & \ddots & \vdots \\
0 & 0 & \cdots & B
\end{array}\right]
$$

Putting (7) and (8) into (4) yield the the matrix form of $U_{n, n}^{(i, j)}(x, y)$ as

$$
U_{n, n}^{(i, j)}(x, y)=\mathbf{X}(x) \mathbf{B}^{i} \mathbf{D}^{T} \overline{\mathbf{Y}}(y) \overline{\mathbf{B}}^{j} \overline{\mathbf{D}} \mathbf{A}
$$

By substituting (3) and (9) in (1), we obtain fundamental matrix equation as

$$
\left[\mathbf{X}(x) \mathbf{B}^{2} \mathbf{D}^{T} \overline{\mathbf{Y}}(y) \overline{\mathbf{D}}+\mathbf{X}(x) \mathbf{D}^{T} \overline{\mathbf{Y}}(y) \overline{\mathbf{B}}^{2} \overline{\mathbf{D}}\right] \mathbf{A}=G(x, y)
$$

By using the collocation points $\left\{\left(x_{i}, y_{j}\right): 0 \leq i, j \leq n\right\}$ in (9), one obtains a matrix $\mathbf{W}_{(n+1)^{2} \times(n+1)^{2}}$ whose $m$-th row, $1 \leq m \leq(n+1)^{2}$, comes from $\left(x_{k}, y_{l}\right), k=\left[\left|\frac{m}{n+1}\right|\right], l=m-k(n+1)-1$. Similarly, $\mathbf{G}$ is column matrix such as

$$
[\mathbf{G}]_{1 m}=G\left(x_{t}, y_{l}\right), \quad t=\left[\left|\frac{m}{n+1}\right|\right] l=m-t(n+1)-1
$$

Thus, a linear system is obtained as

$$
\mathbf{W A}=\mathbf{G}
$$


By using (3) and (9), matrix relations are obtained for the conditions

$$
\mathbf{C A} \sum_{k=0}^{t} \sum_{i=0}^{1} \sum_{j=0}^{1} a_{i, j}^{k} \mathbf{X}\left(\alpha_{t}\right) \mathbf{B}^{i} \mathbf{D}^{T} \overline{\mathbf{Y}}\left(\beta_{t}\right) \overline{\mathbf{B}}^{j} \overline{\mathbf{D}} \mathbf{A}=\lambda_{t}
$$

respectively. Let us write it given in (12) as, respectively,

$$
\mathbf{C A}=\mathbf{G}_{1}
$$

where $\left[\mathbf{G}_{1}\right]_{t 1}=\lambda_{t}$.Combining $[\mathbf{W}, \mathbf{G}]$ and $\left[\mathbf{C}, \mathbf{G}_{1}\right]$ and yields a new system $[\tilde{\mathbf{W}}, \tilde{\mathbf{G}}]$ :

$$
[\tilde{\mathbf{W}}, \tilde{\mathbf{G}}]=\left[\begin{array}{c}
\mathbf{W}, \mathbf{G} \\
\mathbf{C}, \mathbf{G}_{1}
\end{array}\right]
$$

By using the Gauss elimination method, and them by removing zero rows of augmented matrix $[\tilde{\mathbf{W}}, \tilde{\mathbf{G}}]$, we obtain $[\tilde{\mathbf{W}}, \tilde{\mathbf{G}}]$. If $\tilde{\mathbf{W}}$ is a square matrix, then the unknown matrix A is obtained as

$$
\mathbf{A}=\tilde{\mathbf{W}}^{-1} \tilde{\mathbf{G}}
$$

Otherwise, the collocation points should be changes such that $\operatorname{dim}(\tilde{\mathbf{W}})=(n+1)^{2}$. Also, if the columns of $\tilde{\mathbf{W}}$ are linearly independent, then the matrix A can be calculated by the pseudo inverse method; that is,

$$
\tilde{\mathbf{W}}^{\dagger}=\left(\tilde{\mathbf{W}}^{*} \tilde{\mathbf{W}}\right)^{-1} \tilde{\mathbf{W}}^{*}
$$

where $\tilde{\mathbf{W}}^{*}$ is the transpose of $\tilde{\mathbf{W}}$.

\section{Accuracy of the solution and error analysis}

We can easily check the accuracy of the method. Since the truncated Bernstein series (3) is an approximate solution of Eq.(1), when the function $U(x, y)$ and its derivatives are substituted in Eq.(1), the resulting equation must be satisfied approximately; that is, for $(x, y)=\left(x_{q}, y_{q}\right) \in\left\{0 \leq x_{q} \leq a, 0 \leq y_{q} \leq b\right\} q=0,1,2, \ldots E\left(x_{q}, y_{q}\right)=\left|D\left(x_{q}, y_{q}\right)-\lambda I\left(x_{q}, y_{q}\right)\right| \cong 0$ and $E\left(x_{q}, y_{q}\right) \leq 10^{-k_{q}}$ ( $k_{q}$ positive integer). If max $10^{-k_{q}}=10^{-k}$ ( $k$ positive integer) is prescribed, then the truncation limit $N$ is increased until the difference $E\left(x_{q}, y_{q}\right)$ at each of the points becomes smaller than the prescribed $10^{-k}$. On the other hand, the error can be estimated by the function

$$
E_{N}=\sum_{r=0}^{N} \sum_{s=0}^{N} a_{r, s} T_{r, s}(x, y)-g(x, y)-I(x, y)
$$

If $E_{N}(x, y) \rightarrow 0$ when $N$ is sufficiently large enough, then the error decreases.

\section{Numerical example}

In this section, an illustrative example is presented. We have performed numerical computation a computer program written in Maple.

\subsection{Example}

A thing cut-plate homogeneous thermally conducting plate lies in the $x y$-plane and occupies the cut-ring with $|z|=10^{2 K}$ and $|z|=1$. The faces of the plate are insulated and no internal sources or sinks are present. The boundaries of the inner 
periphery of the cut-ring $100^{\circ} \mathrm{C}$ and the boundaries of the outer periphery of the ring $0^{\circ} \mathrm{C}$. The problem is to find the steady temoperature $U(x, y)$ in the plate, the steady state temoperature $U$ must satisfy

$$
U_{x x}(x, y)+U_{y y}(x, y)=0\left(1<x^{2}+y^{2}<10^{2 K}\right)
$$

The boundary conditions are

$$
\begin{array}{r}
U\left(x,+\sqrt{10^{2 K}-x^{2}}\right)=0^{\circ} C U\left(x,-\sqrt{10^{2 K}-x^{2}}\right)=100^{\circ} C\left(1<x<10^{2 K}\right) \\
U\left(x,+\sqrt{1-x^{2}}\right)=U\left(x,-\sqrt{1-x^{2}}\right)=0^{\circ} C\left(1<y<10^{2 K}\right)
\end{array}
$$

We can solving, the conformal mapping of the cut-ring in the $z$-plane onto the rectangle in the $w$-plane can be written as (Fig.3).

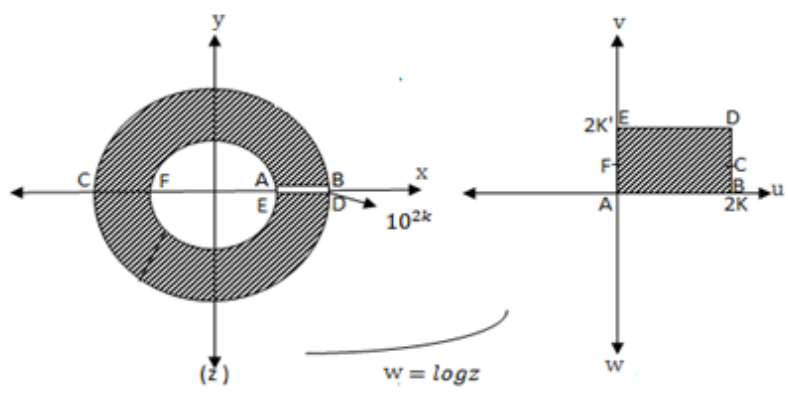

Fig. 3

$\left(|z|<10^{2 K} \wedge|z|<1\right) k=3-\sqrt{2}, K^{\prime}=2 K$ and $K=1,582760 K^{\prime}=3,155879 \cong \pi$ According to this problem, we will get back to conformal mappings.

If the problem is rewritten, (19) is obtained

$$
\begin{array}{rr}
U_{x x}+U_{y y}=0 \quad(0<x<2 K, & 0<y<4 K) \\
U(x, 0)=0^{\circ} C U(x, 4 K)=100^{\circ} C & (0<x<2 K) \\
U(2 K, y)=U(0, y)=0^{\circ} C & (0<y<4 K)
\end{array}
$$

And then the fundamental matrix equation for (19) is obtained as

$$
\left[\mathbf{X}(x) \mathbf{B}^{2} \mathbf{D}^{T} \overline{\mathbf{Y}}(y) \overline{\mathbf{D}}+\mathbf{X}(x) \mathbf{D}^{T} \overline{\mathbf{Y}}(y) \overline{\mathbf{B}}^{2} \overline{\mathbf{D}}\right] \mathbf{A}=0
$$

Let the collocation points be the Chebyshev interpolation nodes

$$
\left\{\left(x_{i}, y_{j}\right): 0<i, j<n, x_{i}=\frac{1}{2}+\frac{1}{2} \cos \left(\frac{2 i-1}{2 n}\right) \pi, y_{j}=\frac{1}{2}+\frac{1}{2} \cos \left(\frac{2 i-1}{2 n}\right) \pi\right\}
$$

or equidistant nodes. Then, $\mathbf{W}$ is a matrix rows are

$$
\mathbf{X}\left(x_{i}\right) \mathbf{B}^{2} \mathbf{D}^{T} \overline{\mathbf{Y}}\left(y_{j}\right) \overline{\mathbf{D}}+\mathbf{X}\left(x_{i}\right) \mathbf{D}^{T} \overline{\mathbf{Y}}\left(y_{j}\right) \overline{\mathbf{B}}^{2} \overline{\mathbf{D}}
$$


and $\mathbf{G}$ is a zero matrix. The condition matrices for $U(x, 0)=0^{\circ} \mathrm{C}, U(x, 4 K)=100^{\circ} \mathrm{CU}(2 \mathrm{~K}, y)=0^{\circ} \mathrm{C}, U(0, y)=0^{\circ} \mathrm{C}$ are obtained as

$$
\begin{aligned}
U_{n, n}\left(x_{i}, 0\right) & =\mathbf{X}\left(x_{i}\right) \mathbf{D}^{T} \overline{\mathbf{Y}}(0) \overline{\mathbf{D}} \mathbf{A}=0 & & i=0,1, . ., n \\
U_{n, n}\left(x_{i}, 4 K\right) & =\mathbf{X}\left(x_{i}\right) \mathbf{D}^{T} \overline{\mathbf{Y}}(4 K) \overline{\mathbf{D}} \mathbf{A}=100 & & i=0,1, . ., n \\
U_{n, n}\left(0, y_{j}\right) & =\mathbf{X}(0) \mathbf{D}^{T} \overline{\mathbf{Y}}\left(y_{j}\right) \overline{\mathbf{D}} \mathbf{A}=0 & & i=0,1, . ., n \\
U_{n, n}\left(2 K, y_{j}\right) & =\mathbf{X}(2 K) \mathbf{D}^{T} \overline{\mathbf{Y}}\left(y_{j}\right) \overline{\mathbf{D}} \mathbf{A}=100 & & i=0,1, . ., n
\end{aligned}
$$

Combining these matrices gives the augmented matrix $[\tilde{\mathbf{W}}, \tilde{\mathbf{G}}]$. By calculating the coefficient matrix $\mathbf{A}$, Bernstein series solutions are obtained for different $n$ values.

for $N=5$

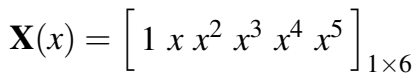

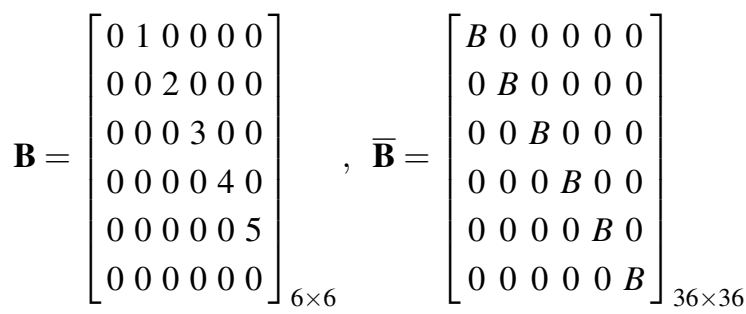

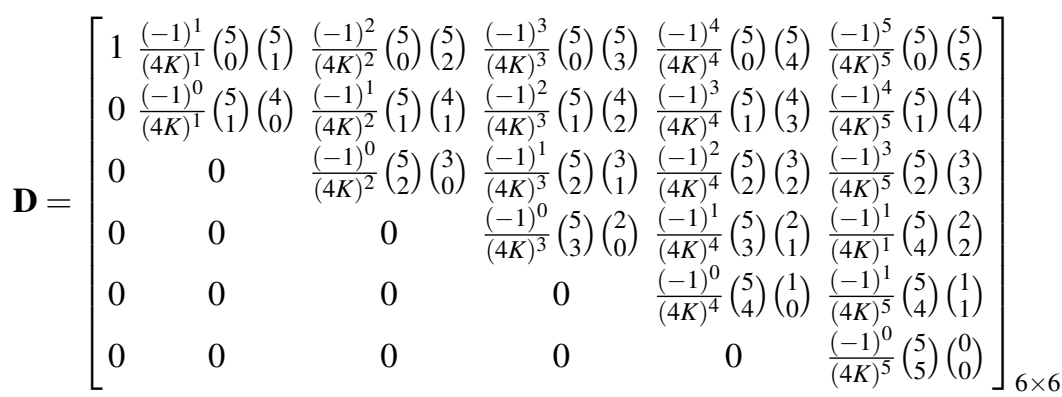

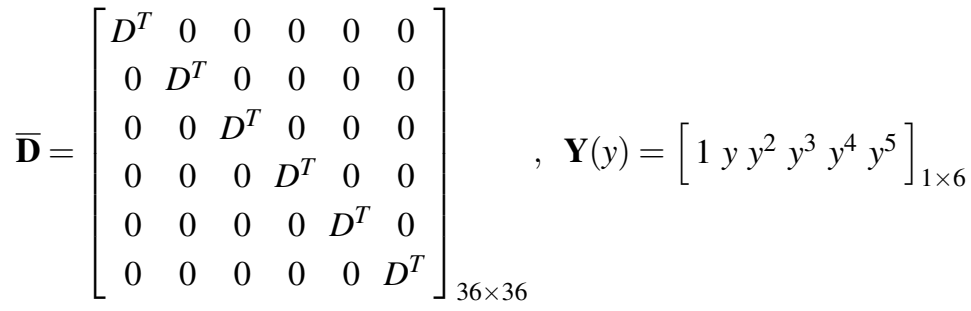

$$
\begin{aligned}
& \overline{\mathbf{Y}}(y)=\left[\begin{array}{cccccc}
Y(y) & 0 & 0 & 0 & 0 & 0 \\
0 & Y(y) & 0 & 0 & 0 & 0 \\
0 & 0 & Y(y) & 0 & 0 & 0 \\
0 & 0 & 0 & Y(y) & 0 & 0 \\
0 & 0 & 0 & 0 & Y(y) & 0 \\
0 & 0 & 0 & 0 & 0 & Y(y)
\end{array}\right]_{6 \times 36}
\end{aligned}
$$

and then, as a result, we get the following error analysis graphics and tables. 


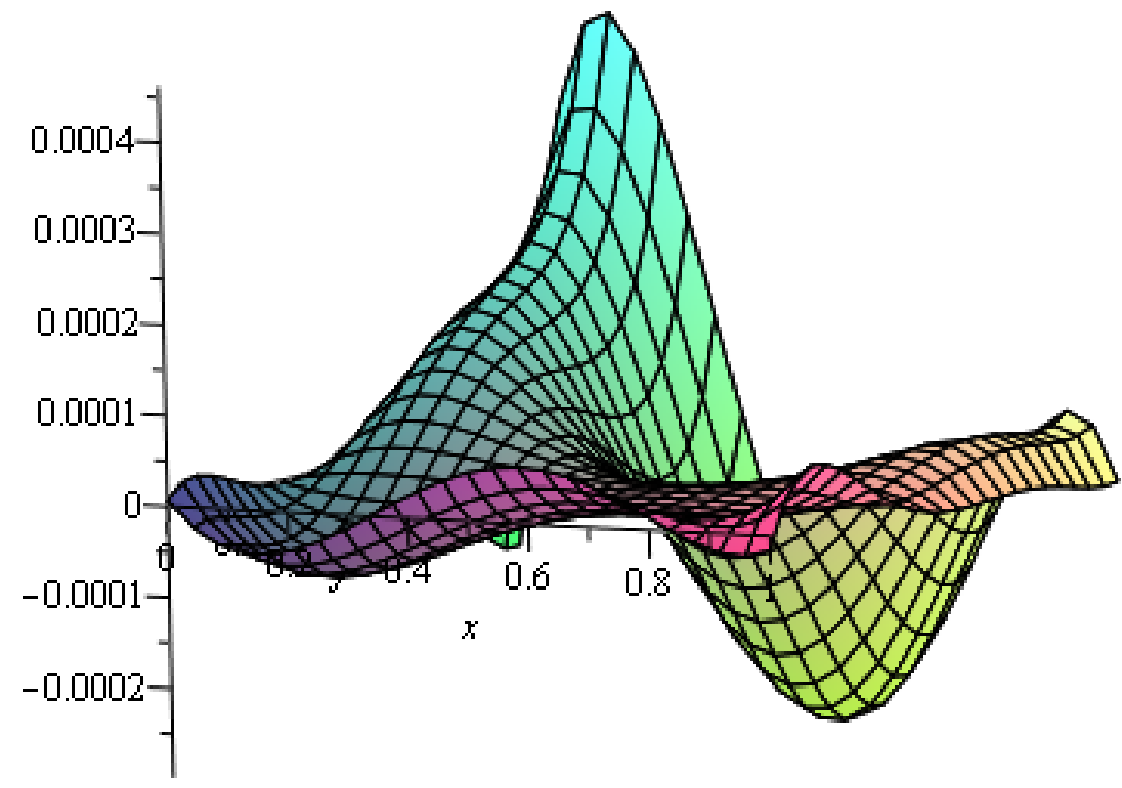

Fig. 4: Error analysis for $N=5$.

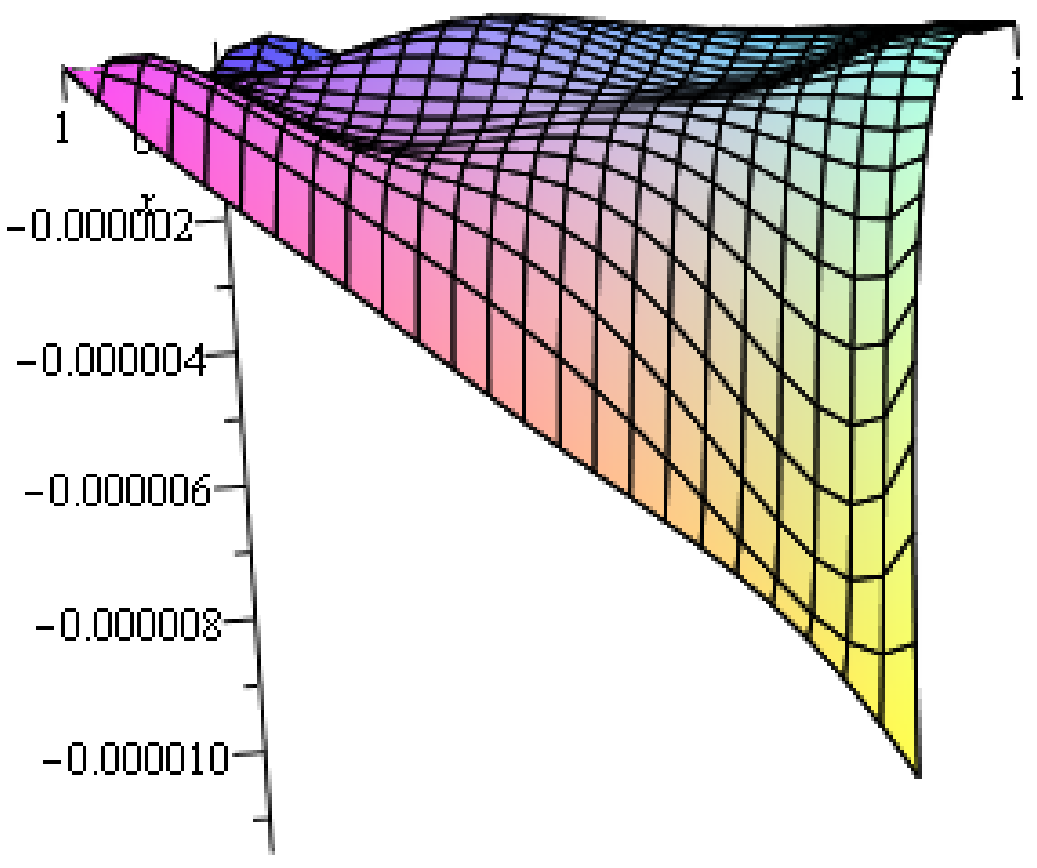

Fig. 5: Error analysis for $N=7$. 


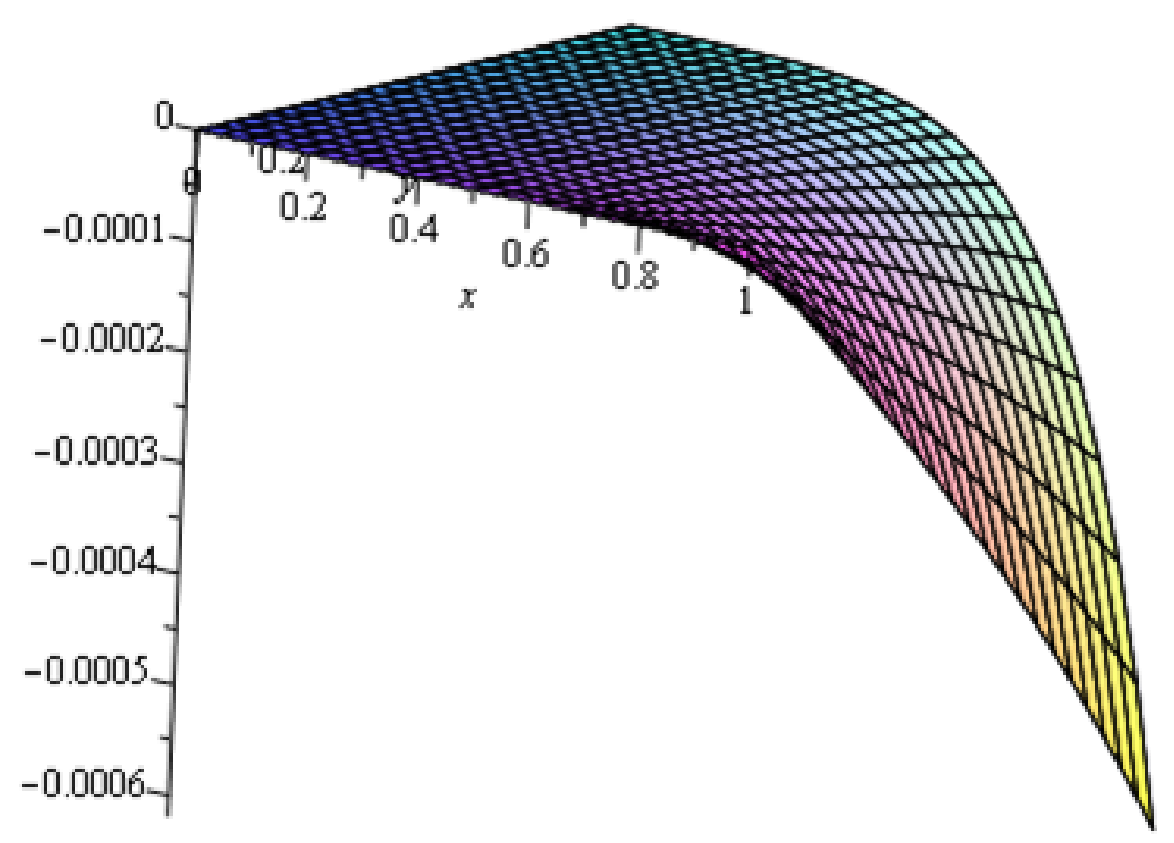

Fig. 6: Error analysis for $N=9$.

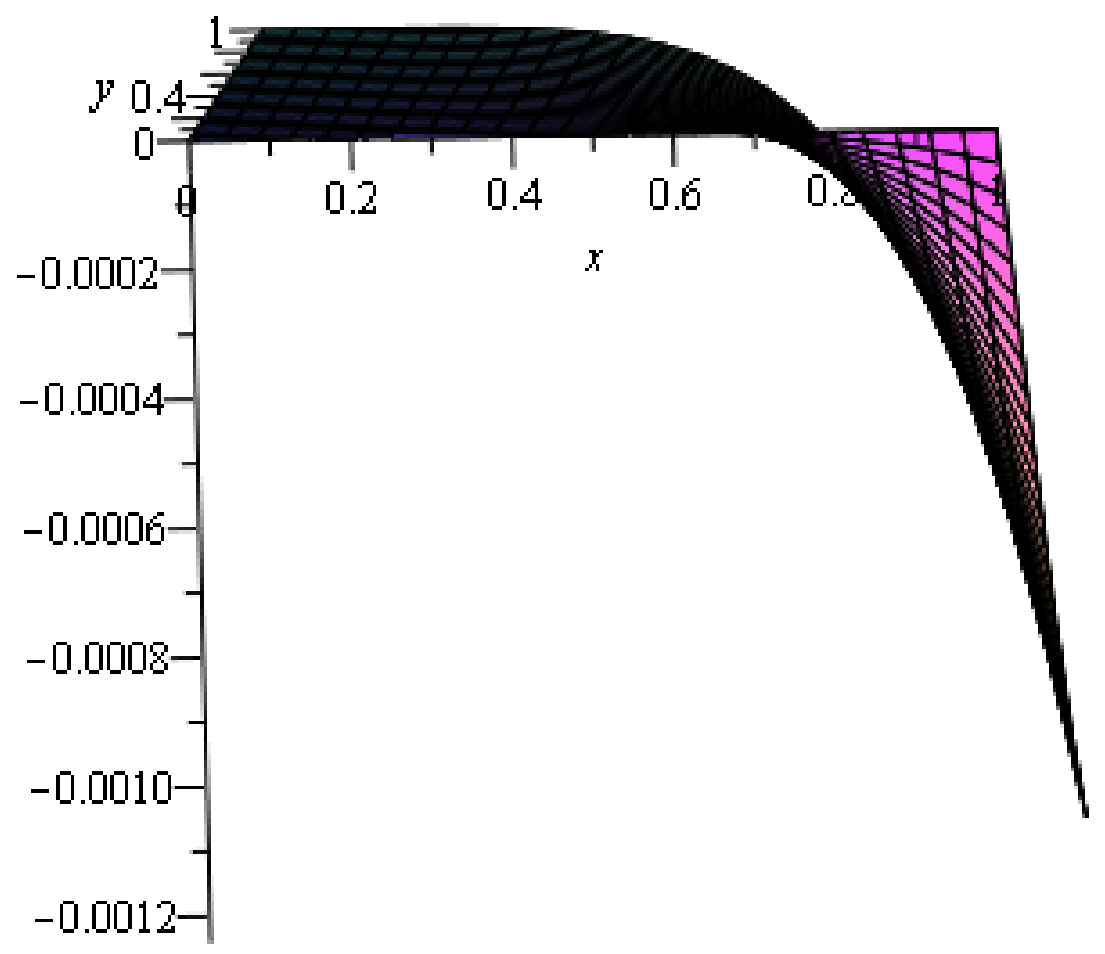

Fig. 7: Error analysis for $N=10$. 


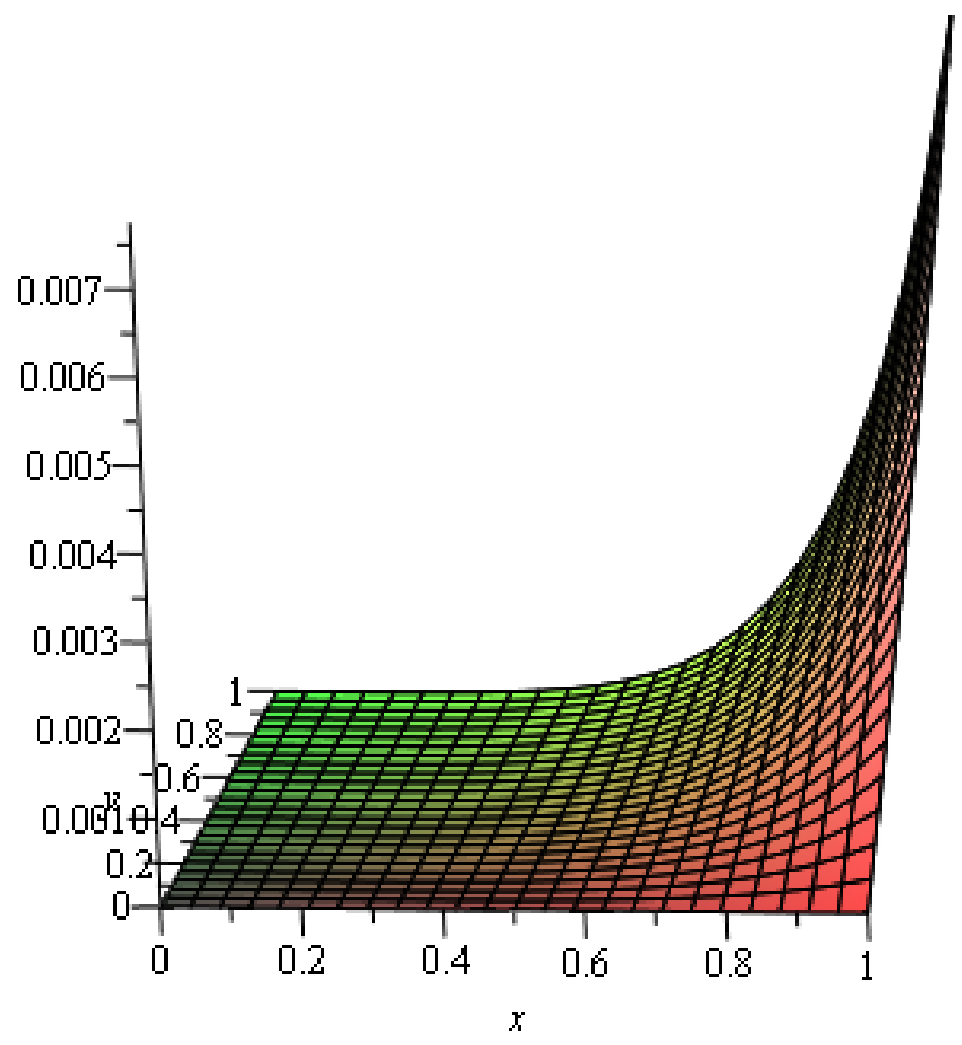

Fig. 8: Error analysis for $N=12$.

Table 1: Comparison of the error analysis on $\partial D$ that is the boundary of $D$ for different values of $N$ for example. $D:(0<x<2 K, 0<y<4 K)$

\begin{tabular}{|c|c|c|c|c|c|c|}
\hline$x$ & $y$ & $N=5$ & $N=7$ & $N=9$ & $N=10$ & $N=12$ \\
\hline 0 & 1 & $-1.315310^{-4}$ & $8.770010^{-9}$ & $-1.536710^{-8}$ & $1.148510^{-9}$ & $-2.599010^{-8}$ \\
\hline 0 & 0.9 & $-1.106910^{-4}$ & $2.515210^{-8}$ & $-8.261210^{-9}$ & $-7.386510^{-9}$ & $-8.630010^{-9}$ \\
\hline 0 & 0.8 & $6.991710^{-6}$ & $4.367210^{-8}$ & $-8.174010^{-11}$ & $-8.502410^{-13}$ & $5.695910^{-15}$ \\
\hline 0 & 0.7 & $-5.959610^{-6}$ & $-1.741210^{-7}$ & $2.331010^{-10}$ & $-7.426010^{-11}$ & $-1.650810^{-8}$ \\
\hline 0 & 0.6 & $1.424510^{-5}$ & $-2.556310^{-7}$ & $6.238410^{-9}$ & $1.385810^{-9}$ & $-1.850110^{-8}$ \\
\hline 0 & 0.5 & $1.142210^{-5}$ & $-1.556610^{-7}$ & $1.301010^{-8}$ & $-6.445910^{-9}$ & $-1.243910^{-8}$ \\
\hline 0 & 0.4 & $4.161410^{-7}$ & $2.602010^{-8}$ & $2.758910^{-10}$ & $-2.753610^{-9}$ & $-1.318010^{-8}$ \\
\hline 0 & 0.3 & $2.781310^{-6}$ & $2.478110^{-8}$ & $-5.894210^{-9}$ & $-4.624110^{-9}$ & $6.750310^{-9}$ \\
\hline 0 & 0.2 & $9.221010^{-6}$ & $-1.696210^{-7}$ & $1.906910^{-8}$ & $-5.118110^{-10}$ & $-3.322810^{-9}$ \\
\hline 0 & 0.1 & $2.548310^{-5}$ & $-2.617210^{-7}$ & $-1.203110^{-9}$ & $-2.818810^{-10}$ & $-1.602010^{-9}$ \\
\hline 0 & 0 & $6.991710^{-6}$ & $4.367210^{-8}$ & $-8.174010^{-11}$ & $-8.502410^{-13}$ & $5.695910^{-15}$ \\
\hline
\end{tabular}

From Table 1, it is obvious that the results get better as $N$ increase. 
Table 2: Comparison of the error analysis in domain $D:(0<x<2 K, 0<y<4 K)$ for $N=5,7,9,10,12$.

\begin{tabular}{|c|c|c|c|c|c|c|}
\hline$x$ & $y$ & $N=5$ & $N=7$ & $N=9$ & $N=10$ & $N=12$ \\
\hline 1 & 1 & $-2.833410^{-5}$ & $-9.700010^{-6}$ & $-3.773210^{-4}$ & $-2.303610^{-3}$ & $4.000010^{-4}$ \\
\hline 0.5 & 0.5 & $-2.144310^{-6}$ & $-3.841010^{-7}$ & $-9.304610^{-6}$ & $-3.137910^{-5}$ & $1.640710^{-4}$ \\
\hline 0.2 & 0.8 & $-4.270610^{-5}$ & $-1.508110^{-7}$ & $-1.767710^{-11}$ & $-1.234610^{-11}$ & $-5.483310^{-15}$ \\
\hline 0.1 & 0.7 & $1.654410^{-4}$ & $1.108610^{-7}$ & $-3.008110^{-8}$ & $-3.919010^{-8}$ & $-1.239910^{-7}$ \\
\hline 0.6 & 0.6 & $-4.500410^{-5}$ & $-1.284810^{-6}$ & $-2.003410^{-5}$ & $-1.443610^{-4}$ & $3.179310^{-4}$ \\
\hline 0.3 & 0.2 & $-6.476910^{-5}$ & $-5.756210^{-7}$ & $1.880610^{-7}$ & $-6.784510^{-7}$ & $1.809810^{-6}$ \\
\hline 1 & 0.4 & $-5.826110^{-6}$ & $-4.210010^{-6}$ & $-1.422710^{-4}$ & $-9.813410^{-4}$ & $3.670010^{-4}$ \\
\hline 0.8 & 0.3 & $-5.772010^{-6}$ & $-8.000010^{-7}$ & $-5.989510^{-5}$ & $-9.243810^{-5}$ & $5.885610^{-4}$ \\
\hline 0.2 & 0.9 & $2.354410^{-4}$ & $-1.043010^{-7}$ & $-3.592010^{-7}$ & $-4.002610^{-7}$ & $2.698010^{-6}$ \\
\hline 0.5 & 0.7 & $-1.206910^{-4}$ & $-1.207410^{-6}$ & $-1.352410^{-5}$ & $-4.437510^{-5}$ & $2.367110^{-4}$ \\
\hline
\end{tabular}

The some calculating values of the error analysis give in Table 2 that is clearly shown when $N$ values increase, error function values rapidly decrease for $N=5,7,9,10$ and 12 .

\section{Conclusion}

The method of conformal mapping is a more flexible and far-reaching tool for the Laplace equation the plane. In a certain sense conformal mapping provides the analoque for elliptic differential equations of the method of characteristic developed for hyperbolic differential equations [4]. To solve partial differential equations numerically, we introduce a new matrix method depending on Bernstein polynomials and collocation points. Present method provides two main advantes: it is very simple to construct the main matrix equations and it is very easy for computer programming.

\section{Competing interests}

The authors declare that they have no competing interests.

\section{Authors' contributions}

All authors have contributed to all parts of the article. All authors read and approved the final manuscript.

\section{References}

[1] Ahmadi MR, Adibi H. The Chebyshev tau technique for the solution of Laplace's equation. Applied Mathematics and Computation 2007; 184(2):895-900.

[2] Baykuş Savaşaneril N, Delibaş H., Analytic solution for two-dimensional heat equation for an ellipse region. NTMSCI 4, No. 1, 65-70 (2016)

[3] Baykuş Savaşaneril N, Delibaş H., Analytic Solution for The Dirichlet Problem in 2-D Journal of Computational and Theoretical Nanoscience. Vol. 15, 1-5, 2018

[4] G. Moretti, Functions of Complex Variable, Prentice-Hall, NJ, 1964.

[5] Hacıoğlu Z, Baykuş Savaşaneril N. and Hasan Köse, Solution of Dirichlet problem for a square region in terms of elliptic functions, NTMSCI 3, No. 4, 98-103 (2015)

[6] Işık O. R , Sezer M. , Güney Z., Bernstein series solution of linear second-order partial differential equations with mixed conditions, Mathematical Methods in the Applied Sciences, 2013 (wileyonlinelibrary.com)DOI:10.1002/mma.2817 
[7] Kurul E. and Baykuş Savaşaneril N.,Solution of the two-dimensional heat equation for a rectangular plate. NTMSCI 3, No. 4, 76-82 (2015)

[8] Kong W, Wu X. Chebyshev tau matrix method for Poisson-type equations in irregular domain. Journal of Computational and Applied Mathematics 2009; 228(1):158-167.

[9] N. Kurt, M. Sezer, A. Çelik, Solution of Dirichlet problem for a rectangular region in terms of elliptic functions, J. Comput. Math. 81 (2004) 1417-1426.

[10] N. Kurt, M. Sezer, Solution of Dirichlet problem for a triangle region in terms of elliptic functions, Appl.Math. Comput. 182 (2006) 73-78.

[11] N. Kurt, Solution of the two-dimensional heat equation for a square in terms of elliptic functions, Journal of the Franklin Institute, 345(3) 2007 303-317.

[12] P. F. Byrd and M.D.Friedman, Handbook of Elliptic Integrals for Engineers and Physicists, Lange Maxvelland Springer Ltd. London, New York, 1954

[13] Sezer M. Chebyshev polynomial approximation for Dirichlet problem. Journal of Faculty of Science Ege University, Series A 1989; 12(2):69-77.

[14] Yüksel G, Işı k O. R and Sezer M. Error analysis of the Chebyshev collocation method for linear second-order partial differential equations, International Journal of Computer Mathematics, 2014 http://dx.doi.org/10.1080/00207160.2014.966099

[15] Yüksel G. Chebyshev polynomials solutions of second order linear partial differential equations. Ph.D. Thesis, Muğla University, Muğla, 2011. 